\title{
Micro-trayectorias de
}

\section{pensamiento científico y}

colaboración en estudiantes

de primaria interactuando

\section{con un videojuego}

Wicro-trajectories of scientific thinking and collaboration in elementary students interacting with a videogame

(c) $($ ) $(9)$
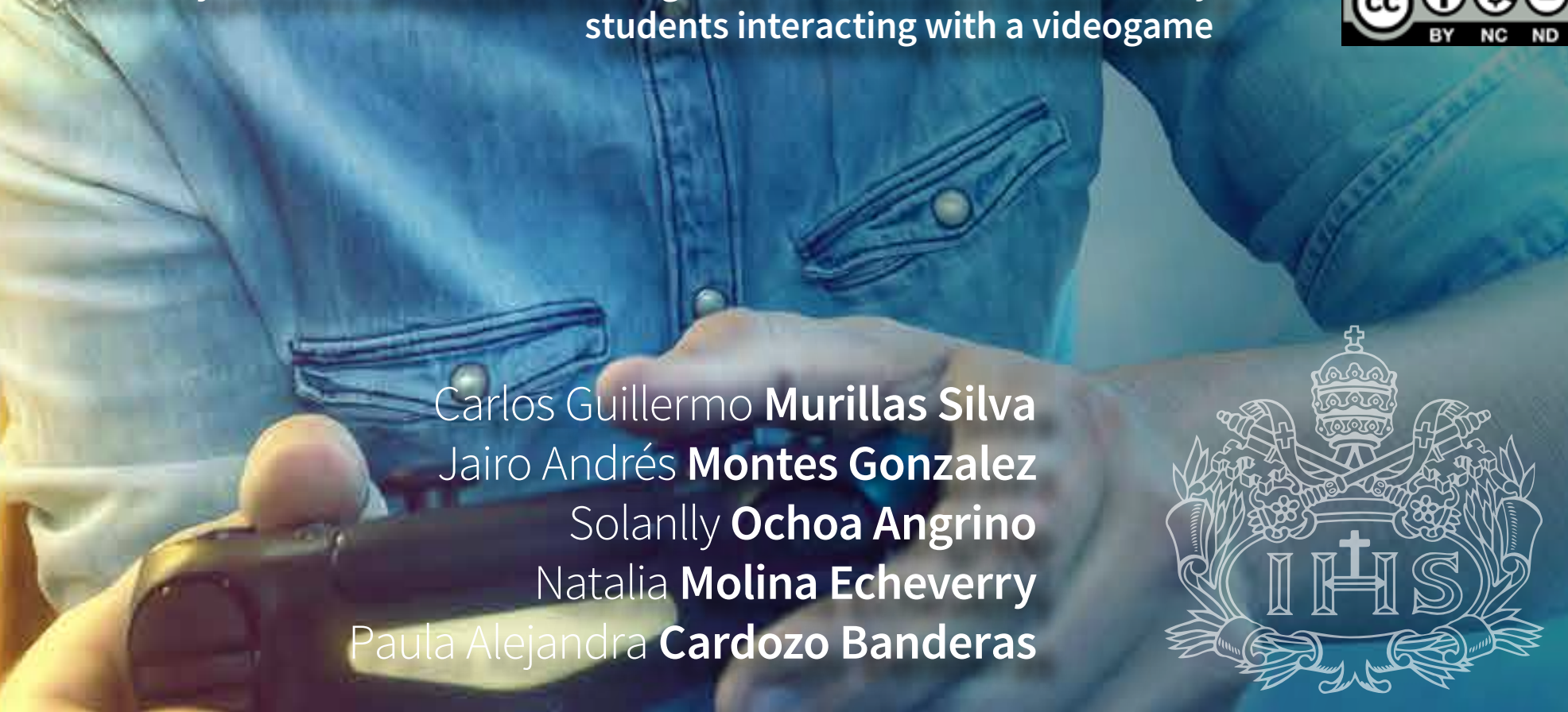

Revista Iberoamericana de

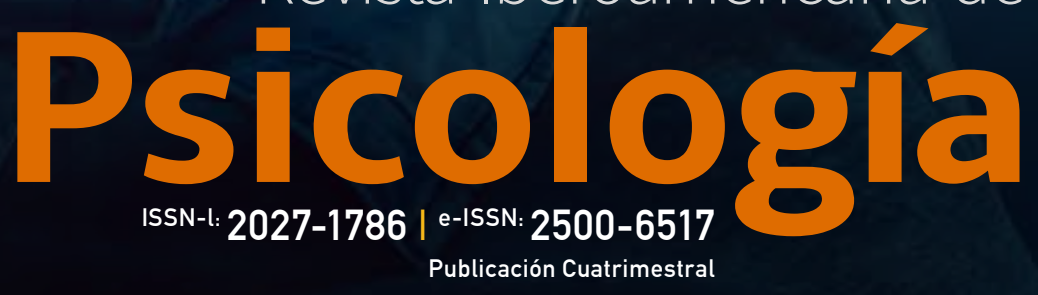




\section{Psicología \\ ISSN-I: 2027-1786 | e-ISSN: 2500-6517} Publicación Cuatrimestral

\section{Solanlly Ochoa Angrino}

ID: 2027-1786.RIP.11303

Title: Micro-trajectories of scientific thinking and collaboration in elementary students interacting with a videogame

Título: Micro-trayectorias de pensamiento científico y colaboración en estudiantes de primaria interactuando con un videojuego

\section{Alt Title / Título alternativo:}

[en]: Micro-trajectories of scientific thinking and collaboration in elementary students interacting with a videogame

[es]: Micro-trayectorias de pensamiento científico y colaboración en estudiantes de primaria interactuando con un videojuego

Author (s) / Autor (es):

Murillas Silva, Montes Gonzales, Ochoa Angrino, Molina Echeverry \& Cardozo Banderas

\section{Keywords / Palabras Clave:}

[en]: Scientific thinking, collaboration, videogames, education, development, microgenesis.

[es]: Pensamiento científico, colaboración, videojuegos, educación, desarrollo, microgénesis

\section{Proyecto / Project:}

No reporta

Submited:

\section{Resumen}

Objetivo: Caracterizar las trayectorias de la colaboración entre estudiantes en segundo y tercer grado de primaria, al resolver un videojuego que demanda pensamiento científico. Método: Se realizó un estudio cuantitativo de diseño microgenético, a través de la aplicación de un videojuego de resolución de problema multivariado durante

9 sesiones a 4 diadas de niños entre 8 y 10 años de edad. Resultados: Se categorizaron las diadas de acuerdo a su colaboración, una diada mostró una tendencia a colaborar, dos diadas oscilaron en los tipos de colaboración (trabajo colaborativo, trabajo paralelo, trabajo

pasivo y no trabajo) y la última diada se concentró en el trabajo pasivo. El desempeño en pensamiento científico varió dependiendo de la categoría de la colaboración, dos diadas mostraron mejora en el desempeño, mientras que en los dos restantes el desempeño osciló

en niveles altos y bajos. Conclusiones: Las diadas que presentaron un tipo de interacción

organizada (trabajo colaborativo y trabajo pasivo) mostraron un mejor desempeño en pensamiento científico, comparadas con las diadas que mostraron una colaboración más oscilante.

\section{Abstract}

Objective: To characterize the trajectories

of collaboration among students of 2 nd

and 3rd grade of elementary school when solving a videogame that demands scientific thinking. Method: A quantitative study

(using microgenetic design) was carried out. Four dyads of children between ages 8 and 10 played during 9 sessions, solving a multivariable problem videogame. Results: The dyads were categorized according to their

level of collaboration: one dyad showed a tendency to collaborate; two dyads oscillated between the following types of collaboration: collaborative work, parallel work, passive work and no-work; the last dyad focused on passive work. Performance in scientific thinking varied depending on the category of collaboration: two dyads showed improvement regarding performance, while the performance of other two oscillated between high and low levels.

Conclusions: The dyads that presented a type of organized interaction (collaborative

work and passive work) showed a better performance in scientific thinking compared to the dyads that showed a more oscillating collaboration.

\section{Citar como:}

Murillas Silva, C. G., Montes Gonzalez, J. A., Ochoa Angrino, S., Molina Echeverry, N., \& Cardozo Banderas, P. A. (2018). Micro-trayectorias de pensamiento científico y colaboración en estudiantes de primaria interactuando con un videojuego. Revista Iberoamericana de Psicología issn-l:2027-1786, 11 (3), 31-46. Obtenido de: https://revistas.iberoamericana.edu.co/index.php/ripsicologia/article/view/1472-5155

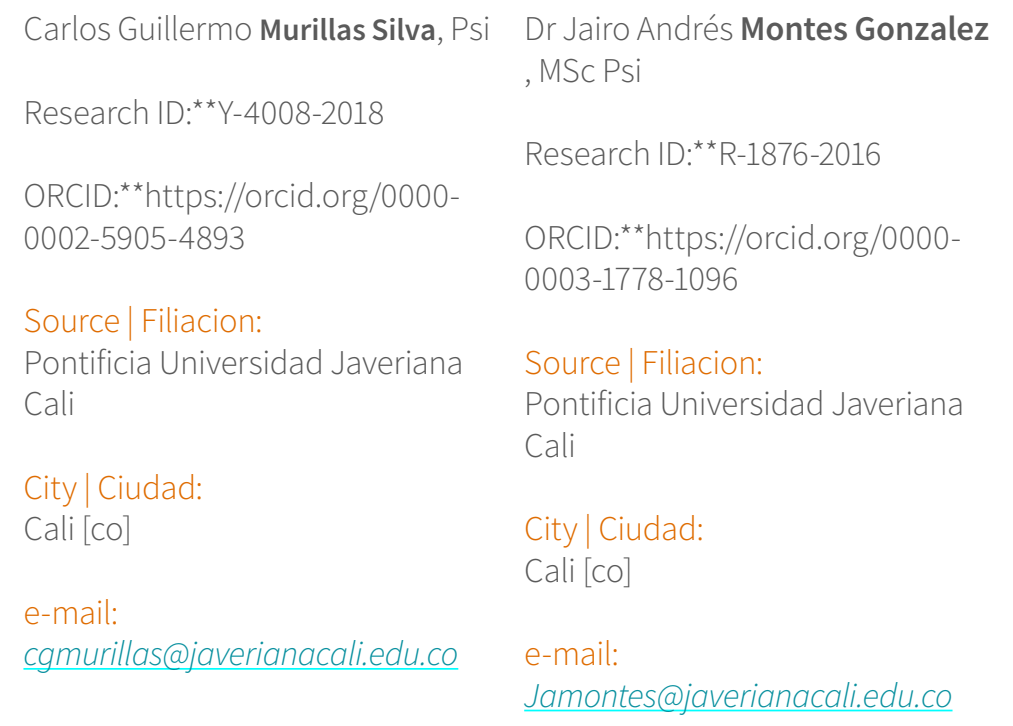

Dra Solanlly Ochoa Angrino, MSc Psi

Research ID: ** N-2005-2016

ORCID:**https://orcid.org/00000002-7043-2808

Source | Filiacion:

Pontificia Universidad Javeriana

Cali

City | Ciudad:

Cali [co]

e-mail:

sochoa@javerianacali.edu.co
Natalia Molina Echeverry, PS

Research ID: ${ }^{\star \star} Y-4506-2018$

ORCID:**https://orcid.org/00000003-0985-4347

Source | Filiacion:

Pontificia Universidad Javeriana Cali

\section{City | Ciudad:}

Cali [co]

e-mail:

natimolina95@javerianacali.edu. CO
Paula Alejandra Cardozo Banderas, Psi

Research ID:**Y-5676-2018

ORCID:**https://orcid.org/0000 0002-7281-0841

Source| Filiacion:

Pontificia Universidad Javeriana Cali

City | Ciudad:

Cali [co]

e-mail:

paulaac@javerianacali.edu.co 


\section{Micro-trayectorias de pensamiento científico y colaboración en estudiantes de primaria interactuando con un videojuego \\ Micro-trajectories of scientific thinking and collaboration in elementary students interacting with a videogame \\ Carlos Guillermo Murillas Silva Jairo Andrés Montes Gonzalez Solanlly Ochoa Angrino Natalia Molina Echeverry Paula Alejandra Cardozo Banderas}

\section{Introducción}

En el 2015 la asamblea general de la ONU trazó los objetivos del desarrollo sostenible, entre los cuales, en cuarto lugar, se encuentra garantizar educación de calidad (Organización de las Naciones Unidas 2015). El cumplimiento de este objetivo cobra importancia para Colombia si se tiene en cuenta que los niveles de educación en nuestro país son bajos, de acuerdo a la Organización para la Cooperación y el Desarrollo Económico (OECD), organización que mide el nivel educativo con base en varios criterios, entre ellos el desempeño en ciencias, matemáticas y lectura. De acuerdo con datos reportados por esta entidad, en el año 2015, Colombia se encontraba considerablemente por debajo del promedio mundial en los tres criterios mencionados, y particularmente en el área de ciencias (Promedio mundial en ciencias, 493, promedio de Colombia, 416).

Estas cifras coinciden con las arrojadas por el Instituto Colombiano para el Fomento de la Educación Superior (2016), entidad gubernamental encargada de evaluar factores que influyen en la educación de los colombianos, dejan en evidencia que el desempeño obtenido por los estudiantes que presentaron las pruebas saber $11^{\circ}$ en el 2015, en las áreas de ciencias (biología, física y química) estuvieron por debajo del promedio (50) con puntajes de 45, 46 y 47 respectivamente.
Del mismo modo, al observar las cifras que arroja el ICFES sobre las pruebas pre saber $3^{\circ}, 5^{\circ}$ y $9^{\circ}$, se encuentra que para el 2014 la mayoría de los estudiantes están en un nivel mínimo.

Este panorama es particularmente preocupante al tener en cuenta que la enseñanza de las ciencias tiene relevancia en la formación de las habilidades para afrontar las demandas del momento histórico. Eso es evidente al revisar los objetivos para el 2030 planteados por la ONU (2017) los cuales dan un marco de referencia sobre las competencias a desarrollar en las próximas décadas; en dichos objetivos, específicamente, se plantea como propósito que los estudiantes sean capaces de comprender temas de salud, alimenticios y energéticos, medio ambientales, medidas físicas, entre otros.

Las investigaciones en educación científica han abordado muchos de estos contenidos, así como la manera como los docentes y estudiantes interactúan con ellos. Algunos estudios han abordado áreas como, la química (Quintanal Pérez, 2016) la física (Anderson \& Barnett, 2013) las matemáticas (Fengfeng, 2008; Brom, Preuss, \& Klement, 2011) y la biología (Brom, Preuss, \& Klement, 2011; Castillo, Checa, García-Varela, Herrero, \& Monjelat, 2014). No obstante, las necesidades de formación e investigación en educación científica van más allá de temas científicos. La educación en las ciencias debería estar direccionada para favorecer el desarrollo de habilidades para 
investigar, comprender y evaluar el contenido científico, sus procesos y productos. Además, debería brindar a los estudiantes herramientas que facilitan la comprensión y una mirada crítica hacia la realidad (Morris, Croker, Zimmerman, Gill, \& Roming, 2013)

Un ejemplo de una perspectiva que favorece las habilidades sobre los contenidos se observa en el estudio de Corredor, Gaydos, \& Squire (2013) quienes realizaron una investigación en la clase de biología de estudiantes de primaria. Esta se llevó a cabo durante 4 semanas, cada una con 4 sesiones semanales. La investigación tuvo como objetivo explorar cómo el videojuego Virulent facilita la habilidad para construir representaciones de fenómenos con dimensiones temporales en el área de la biología. Esta investigación hizo énfasis en el proceso de adquisición de habilidades para la construcción de modelos mentales dinámicos a partir de la observación y protocolos de pensamiento en voz alta, los cuales sirvieron para dar cuenta de la forma como los estudiantes iban desarrollando esta destreza durante el uso del juego. Trabajos semejantes usan las TIC para favorecer procesos de adquisición de diferentes habilidades y conceptos asociados a la física newtoniana (Clark, y otros, 2011), el manejo y administración de recursos naturales (Schulze, y otros, 2015) y procesos biológicos (Neulight, Kafai, Kao, Foley, \& Galas, 2007).

Los anteriores son ejemplos de investigaciones que han surgido en respuesta a la búsqueda de nuevas estrategias de innovación educativa y que han encontrado en las TIC, específicamente en los videojuegos, un gran potencial para el desarrollo y adquisición de habilidades. Tales investigaciones van en la línea de las investigaciones que sugieren que implementar herramientas como los videojuegos puede ofrecer espacios auténticos en los que los estudiantes pueden desarrollar el pensamiento científico (Anderson, y otros, 2009; Squire \& Jan, 2007).

No obstante, presentar los videojuegos como herramientas tecnológicas que pueden llegar a generar procesos de pensamiento científico, implica comprender cómo ellos contribuyen a desarrollar dichas habilidades. Para responder este interrogante, Morris, Croker, Zimmerman, Gill, \& Roming (2013) proponen una taxonomía sobre los diferentes aspectos que son potenciados con el uso de videojuegos; basado en las interacciones que se genera entre estos y sus usuarios, estos autores señalan aportes del uso de videojuegos en tres grandes dominios: motivación, cognición y metacognición.

En cuanto a la motivación, los videojuegos tienen en sus características elementos que los hacen herramientas generadoras de este proceso. Cuando una persona utiliza su videojuego favorito parece disfrutar profundamente, se concentra totalmente, se altera la percepción del tiempo y puede enajenarse de lo que le rodea hasta terminar la actividad. Estas experiencias son descritas por Csikszentmihalyi (2008) como experiencias de flujo y se relacionan con el disfrute de actividades que han sido escogidas por el placer que genera realizarlas. En el caso de los videojuegos, se ha encontrado que los niños deciden usar videojuegos por el gusto de jugarlos más que por una recompensa o estimulo externo (McGonigal, 2011; Przybylski, Ryan, \& Rigby, 2009). Algunas investigaciones que han explorado las aspectos motivacionales y de compromiso, por parte de diferentes usuarios cuando utilizan los videojuegos se pueden encontrar en: (Shernoff, Hamari, \& Rowe, 2014; Kiili, de Freitas, Arnab, \& Lainema, 2012; Papastergiou, 2009; Shernoff, Csikszentmihalyi, Schneider, \& Steele, 2003)

El segundo dominio, el de la cognición, implica el desarrollo de habilidadesempleadasen el pensamiento científico como identificación de problemas, generación de hipótesis, diseño de experiencias, recolección de información y producción de inferencias. Aunque las anteriores actividades demandan procesamiento de pensamiento de alto orden, las habilidades que se requieren para resolver problemas de esa índole no se generan de manera espontánea, razón por la cual es necesario soportarlas con herramientas que andamien su aparición (Morris, Croker, Zimmerman, Gill, \& Roming, 2013). Algunas de las investigaciones que han trabajado estos aspectos basadas en el uso de videojuegos y relacionadas con temas de evolución pueden encontrarse en Castillo, Checa, García-Varela, Herrero, \& Monjelat (2014), asociadas a temas de Salud en Papastergiou (2009), concernientes a temas de Genética en Annetta, Minogue, Holmes, \& Cheng (2009) y específicamente en el campo de la Biología en Brom, Preuss, \& Klement (2011).

El último de los dominios es la metacognición, este proceso se entiende como el pensar sobre el propio pensamiento. Cuando un niño se enfrenta a una tarea o un problema no solamente está pensando, aprende a pensar sobre la tarea, sobre su propio pensamiento, planea estrategias y formula modos de resolver el problema planteado. Este proceso no solo involucra lo que el niño sabe de su conocimiento sino, también, cómo es capaz de dominar eso que sabe (Flavell, Miller, \& Miller, 2002). Así las cosas, al interactuar con el videojuego se generan un andamiaje de las estrategias metacognitivas en el que se busca que el jugador reflexione sobre su proceso de construcción de conocimiento a medida que va avanzando en el dominio del videojuego. En otras palabras, la cantidad y calidad de retroalimentación más avanzada favorece el desarrollo de habilidades en el jugador (Flavell, 1979; Ritterfeld \& Weber, 2006; Torrance, 2007). Varias investigaciones han explorado los efecto sobre los aspectos metacognitivos basados en la implementación de videojuegos (Kim, Park, \& Baek, 2009; Neulight, Kafai, Kao, Foley, \& Galas, 2007; Ko, 2002).

Teniendo en cuenta lo anterior, es posible afirmar que basados en la evidencia, los videojuegos tienen ciertas características que permiten, a partir de las interacciones con el jugador, promover procesos de aprendizajey desarrollo de habilidades cognitivas de orden superior. Sin embargo, es necesario tener en cuenta la complejidad del fenómeno detrás de la promoción de dichos aspectos, por lo que no es posible pensar que los videojuegos por sí solos son capaces de promoverlos, sino que es necesario que su uso se de en condiciones particulares que favorezcan su aparición (Fengfeng, 2008; Lacasa, Martínez, \& Méndez, 2008).

Por otra parte, los videojuegos también encarnan competencias y habilidades de carácter social que promueve la interacción entre los jugadores más que entre el jugador y la "máquina" (Zhu \& Chen, 2012). Partiendo de esto, es posible pensar que una condición que tendría efecto positivo para el uso y aprovechamiento de los videojuegos podría ser el trabajo colaborativo, el cual permitiría la interacción e intercambio de ideas entre dos o más personas y en el que cada miembro haría distintos aportes que permitirían que el grupo llegue a la meta o la tarea esperada (Kuhn, 2015). La evidencia sugiere que la colaboración entre pares facilita la construcción de conocimiento por medio del intercambio de los puntos de vista y la argumentación. En este sentido, el aprendizaje centrado en la colaboración ha sido considerado como una de las fuentes fundamentales para promover procesos de aprendizaje y favorecer la resolución de problemas, mostrando resultados mejores de los obtenidos cuando se trabaja a nivel individual, (Koriat, 2012)

Al explorar sobre la colaboración y el uso de videojuegos, se encuentra que las investigaciones se alinean con la idea según la cual los estudiantes que hacen uso de un videojuego en interacción 
tienen mejores resultados que al trabajar individualmente. Lo anterior, no toma en cuenta la complejidad del fenómeno y lo asume como una propiedad que está o no en la sesión de juego. Por ejemplo, Sung \& Hwang (2013) exploraron el cambio de la motivación hacia el aprendizaje, las actitudes hacia la ciencia y la autoeficacia cuando se emplea un videojuego en parejas e individualmente, encontrando que los grupos que jugaron en parejas obtuvieron mayores puntajes en las tres categorías.

Así mismo, Sánchez, Mendoza, \& Salinas (2009) realizaron otra investigación cuyo objetivo fue evidenciar el impacto del trabajo colaborativo entre pares al resolver un problema presentado a través de un videojuego, encontrando que los estudiantes que resolvieron la tarea en parejas lograron progresar a niveles avanzados del juego y mostraron mayor participación durante el proceso, a diferencia de los estudiantes que lo hicieron individualmente. En esta misma línea, al indagar por investigaciones en el tema en niños de edades más avanzadas, Fawcett \& Garton (2005) encontraron que los efectos de la colaboración no son completamente claros; en su investigación encontraron que estudiantes de 7 años trabajando juntos obtenían mejores resultados que los obtenidos individualmente. Sin embargo, notaron también que los efectos de la colaboración variaban dependiendo de la habilidad de los estudiantes; así, los estudiantes que más se beneficiaban de la colaboración fueron los de menor habilidad, mientras que los que colaboraban y eran hábiles en la tarea no se vieron tan beneficiados (Fawcett \& Garton, 2005).

No obstante, esta conclusión con relación al efecto positivo de la colaboración en la comprensión y el desempeño es cuestionada por otros trabajos. Por ejemplo, las investigaciones en interacciones han encontrado que, en la interacción de dos sujetos puede o no presentarse la colaboración (Kuhn, 2015; Guevara, Dijk, \& Geert, 2016). Además, diferentes estudios han encontrado que, cuando niños de temprana edad se enfrentan a tareas de resolución de problemas se presenta poca colaboración. En esta línea Guevara, Dijk, \& Geert (2016) encontraron que niños entre 4 y 6 años, durante una actividad de resolución de problemas en parejas, principalmente tomaban turnos y trabajaban independientemente. De acuerdo con sus hallazgos solo en pocas ocasiones los estudiantes mostraron colaborar entre sí, ni siquiera en aquellas situaciones donde la dificultad se incrementaba.

No obstante, la perspectiva anterior puede resultar limitante a la hora de explorar el tema de los videojuegos, principalmente debido a que no toma en cuenta el carácter dinámico del fenómeno de la interacción en el tiempo de juego. A partir de lo anterior, convendría entonces reconocer que el trabajo colaborativo se presenta como un fenómeno complejo, dinámico y variable, el cual está relacionado con la coexistencia y relación de múltiples factores que hacen posible o no la emergencia del mismo y el posible favorecimiento de mejores desempeños y aprendizajes en los estudiantes. Entre estos factores están: la habilidad de los sujetos de resolver problemas (Golbeck, 1998), la diferencia de los materiales de aprendizaje (Mullins, Rummel, \& Spada, 2011), las habilidades de interacción de las diadas (Park \& Lee, 2015), el dominio de una tarea en específico y la diferente en las habilidades entre la diada (Sills, Rowse, \& Emerson, 2016). Adicionalmente, lo anterior se relaciona con limitaciones de tipo metodológico, teniendo en cuenta el predominio de diseños transaccionales con grupo control, que evalúan el "efecto" del videojuego y/o la presencia de la interacción (Cimpian, 2010; Plass, y otros, 2013; Sung \& Hwang, 2013), pero no se hacen evidentes los procesos de desarrollo de las diferentes habilidades que emergen al jugar en tiempo real.

Una alternativa que surge en respuesta de esta limitante metodológica es el abordaje microgenético, el cual busca comprender los procesos de desarrollo de un sujeto y permite medirlos a través del tiempo. Este abordaje ha sido implementado principalmente para estudiar el desempeño de niños en distintas situaciones de aprendizaje (Guevara, Dijk, \& Geert, 2016). Esta alternativa metodológica también se ha empezado a explorar en la interacción con videojuegos (Anderson, y otros, 2009; Shute \& Kim, 2012).

Partiendo de la orientación microgenética y de su aplicación al estudio de los videojuegos, este artículo busca responder a las preguntas: ¿Cómo se presentan las trayectorias de interacción de diadas al resolver un problema científico a manera de videojuego? Y ¿Cuál es la relación entre las interacciones de los estudiantes al jugar en diadas y su desempeño durante múltiples sesiones al resolver un problema científico a manera de videojuego? La propuesta metodológica para el abordaje de estas preguntas, así como los resultados obtenidos se presentan a continuación.

\section{Método}

El estudio tuvo un diseño microgenético, el cual es definido por Kuhn (2015) como un método donde se observa el desempeño de un individuo en una actividad durante múltiples sesiones, es decir, los participantes son observados durante el proceso de adquisición de nuevos conocimientos o habilidades en el transcurso del tiempo. Este diseño permite la externalización visible del desarrollo de las representaciones internas que el individuo construye durante la realización de una actividad. Debido a que toda actividad es un proceso que puede ocurrir en segundos, horas o días (Bermejo, 2005).

El método microgenético permite comprender el cambio cognitivo que ocurre en casos particulares, debido a que este busca observar individualmente a cada niño durante el tiempo en el que ocurren los cambios cognitivos. Este método también involucra una alta cantidad de observaciones en relación con los periodos de cambio y se hace énfasis en el análisis de caso por caso para lograr inferir cuales son los procesos que llevan a generar el cambio cognitivo (Siegler, 1994). Adicionalmente, este método de investigación también considera la variabilidad de los fenómenos, ya que el alto número de observaciones en periodos prolongados de tiempo que pueden ir de varias semanas hasta meses, aumenta la posibilidad de observar el cambio cognitivo y la variedad de estrategias cognitivas que emplean los niños al tratar de resolver un problema (van Geert y van Dijk, 2002).

Desde esta perspectiva, la generalización de resultados consiste en realizar descripciones detalladas de múltiples mediciones en un mismo sujeto y a diferencia de otros métodos afines, no se da a través de datos de una muestra representativa con pocas medidas para cada sujeto estudiado. En este sentido, se puede llegar a descripciones detalladas que pueden llegar a convertirse en generalizaciones, a partir de la construcción de un modelo con base a las principales interacciones, procesos y componentes que ocurren durante el cambio cognitivo en cada caso (Molenaary Valsiner, 2005).

\section{Participantes}

Ocho estudiantes de segundo y tercer grado de un colegio privado de la ciudad de Cali, Colombia, de edades entre ocho y diez años, quienes se dividieron en cuatro diadas para interactuar con el videojuego. El muestreo con el que se seleccionaron a los participantes fue no probabilístico y se realizó a conveniencia de los investigadores, debido a que los sujetos de estudio son accesibles y adecuados a las 


\section{Micro-trayectorias de pensamiento científico y colaboración en estudiantes de primaria interactuando con un videojuego}

condiciones de trabajo de los investigadores (McMillan \& Schumacher, 2005).

\section{Instrumento}

Los datos se recolectaron a partir del juego “Terapeia”, el cual está diseñado bajo las características de una herramienta que promueve el aprendizaje de contenido en ciencias naturales, pero, sobre todo, el desarrollo del pensamiento científico. Este desarrollo implica el uso de capacidades complejas de resolución de problemas que permiten al jugador identificar relaciones entre variables, predecir el resultado de interacciones entre éstas y distinguirlas de otras no causales.

Dentro del juego, el jugador asume el rol de médico, quien debe resolver diferentes casos enfrentando situaciones concretas de enfermedades (16 cuadros clínicos) y solucionar problemas de salud. Estos problemas implican que decida en tiempo real cual puede ser la mejor manera de restablecer la salud del paciente; por medio de la manipulación de variables como medicamentos, (antibióticos, antihistamínicos, antimicóticos) y de la consideración de factores de riesgo o protección (alimentación, contacto con alergénicos, ejercicio.) del paciente. Estos cuadros clínicos estuvieron distribuidos en tres niveles de dificultad, los cuales variaron de acuerdo al número de variables implicadas, su combinación e impacto en el paciente. Cabe resaltar que en cada nivel se presentaron diferentes cuadros clínicos a resolver y la mayor parte de éstos después del nivel dos fueron
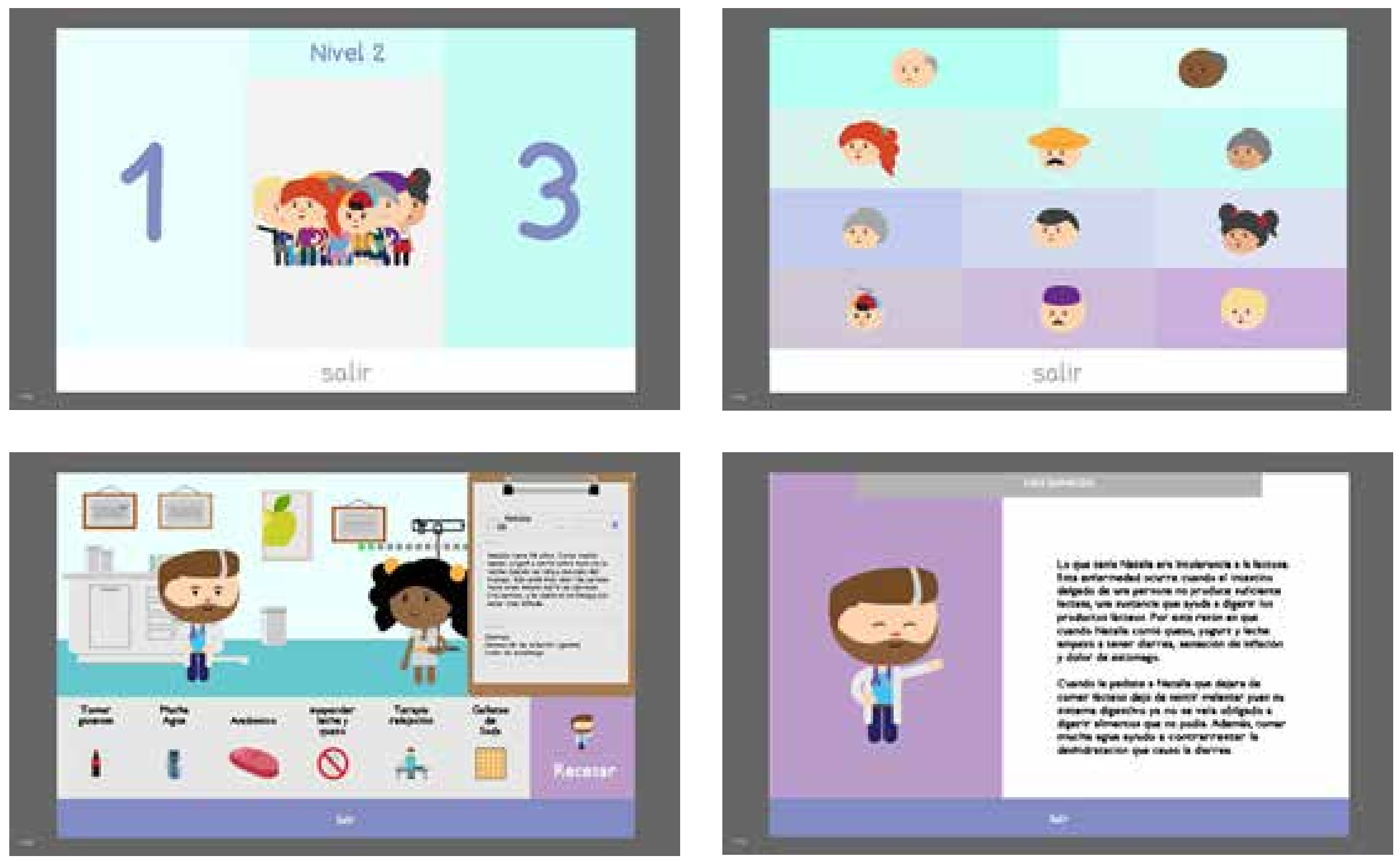

comunes para dos o tres pacientes, lo cual abre igual número de casos a resolver, pero demandan el uso de diferentes tratamientos y, por ende, diferentes combinaciones de variables.

El juego Terapeia se compone de tres niveles de dificultad, el primer nivel se compone de cuatro casos independientes, en el primer caso, el paciente presenta un orzuelo, en el segundo presenta acné, en el tercero presenta fatiga y debilidad muscular y en el último de este nivel, presenta un resfriado. El segundo nivel se compone de cuatro problemáticas, la primera presenta dos casos de pacientes distintos, sin embargo, la problemática por la que ambos acuden es el dolor en el pecho, dificultad para respirar y fatiga. La segunda problemática de este nivel se compone de tres casos, en donde los pacientes acuden con debilidad en los músculos y fatiga, la tercera problemática se compone también de tres casos y los pacientes acuden con dolor en las rodillas y dificultad para caminar, la cuarta y última problemática de este nivel se compone también de tres casos, en donde los pacientes acuden con dolor al orinar, dolor lumbar, cólico y fiebre.

El tercer nivel se compone de cinco problemáticas, la primera de ellas tiene dos casos donde los pacientes acuden con tos seca y con flema, fiebre y respiración con silbido, la segunda problemática se compone de tres casos donde los pacientes acuden con diarrea, sensación de inflamación (gases) y dolor de estómago. En la tercera problemática los pacientes acuden con cansancio/fatiga, sed, aumento de peso y van muchas veces al baño, en la cuarta problemática los pacientes presentan problemas en el colegio, irritabilidad, falta de concentración y ansiedad, y en la última problemática, los pacientes presentan erupciones, piel enrojecida, adolorida y piel inflamada.

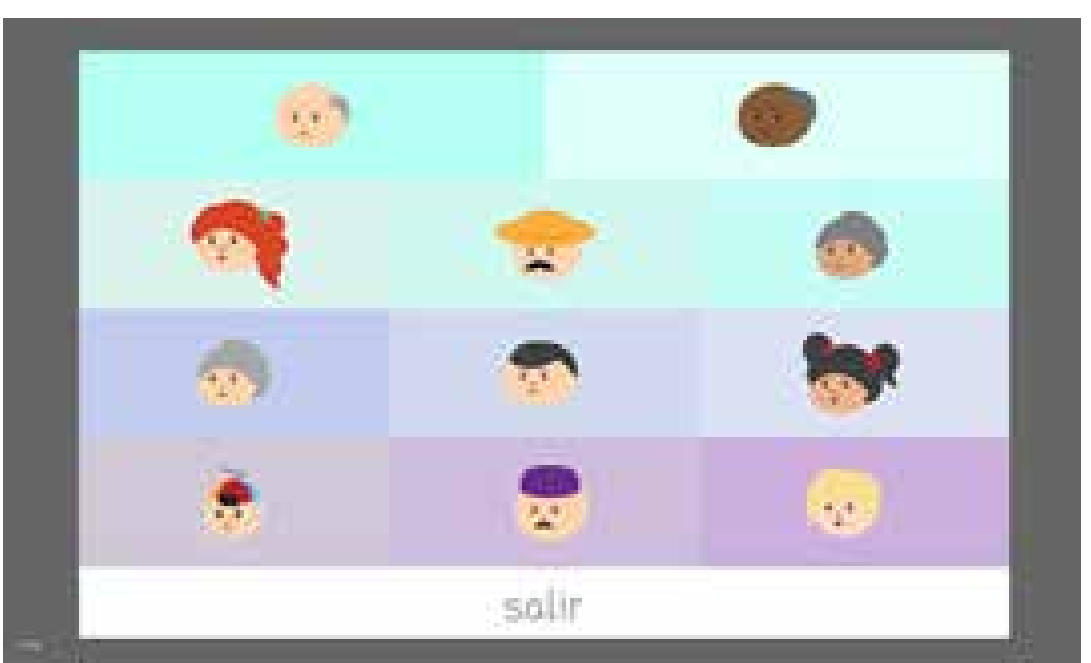

Figura 1, se observa la interfaz del juego, en ellas se ve uno de los casos que se presenta a los jugadores y las diferentes opciones de tratamiento a su disposición.

Figura 1. Interfaz del videojuego "Terapeia" 


\section{Técnica de codificación de las interacciones}

La interacción de las diadas con la tarea se codificó de acuerdo a la participación activa de cada sujeto con la misma y con su compañero. Cada intento que la diada realizó para intentar curar la enfermedad de su paciente representó una unidad de análisis.

Para caracterizar las interacciones de las diadas se tuvo como base las categorías deinteracción planteadas por Guevara, Dijk, \& Geert (2016) quien propone cuatro formas de interacción: a) trabajo colaborativo, donde ambos participantes trabajan activamente y conjuntamente para resolver el problema, b) trabajo paralelo, donde ambos trabajan para resolver la tarea pero lo hacen independientemente, c) trabajo pasivo donde uno de los participantes intenta resolver la tarea mientras el otro observa, y d) No trabajo, donde uno de los participantes trabaja para resolver la tarea mientras que el otro no lo hace, es decir, es totalmente ajeno a la resolución de la tarea (Ver Tabla 1).

Se implementó la técnica rejilla de estado-espacio, en tanto constituye un método que permite graficar la forma como un sistema, es decir, como la diada al interactuar se mueve a partir de dos dimensiones, las cuales se componen de las distintas formas en las que el sistema determinado puede comportarse, de modo, es posible seguir en tiempo real los cambios que este tenga en un periodo de tiempo. (Hollenstein, 2007). De esta forma, por ejemplo, cuando las interacciones de la diada sean colaborativas, en la gráfica aparecerá un círculo azul en el cuadrante superior derecho.

En la Tabla 1 encuentran descritas las categorías de interacción y el cuadrante donde estas se ubicarán.

Tabla 1 Categorías de interacción y cuadrantes de ubicación

\begin{tabular}{|c|c|c|c|}
\hline $\begin{array}{c}\text { Categoría de la } \\
\text { interacción de las } \\
\text { diadas }\end{array}$ & Definición operacional & \multicolumn{2}{|c|}{$\begin{array}{c}\text { Cuadrante de la rejilla de estado- } \\
\text { espacio }\end{array}$} \\
\hline Trabajo colaborativo & $\begin{array}{l}\text { Ambos niños interactúan con la } \\
\text { tarea, tiene en cuenta la opinión } \\
\text { de su compañero y su } \\
\text { comportamiento está encaminada } \\
\text { en realizar la tarea de modo } \\
\text { colaborativa. }\end{array}$ & & 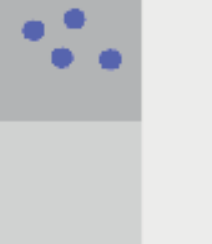 \\
\hline Trabajo Paralelo & $\begin{array}{l}\text { Se tiene interacción con la tarea, } \\
\text { pero se deja a un lado la opinión } \\
\text { del compañero, es decir, los dos } \\
\text { niños trabajan en la misma tarea, } \\
\text { pero lo hacen de forma } \\
\text { independiente. }\end{array}$ & & $0_{0}^{0}$ \\
\hline Trabajo Pasivo & $\begin{array}{c}\text { El niño interactúa con la tarea, } \\
\text { pero no tiene una participación } \\
\text { activa, esté observa mientras el } \\
\text { compañero trabaja. }\end{array}$ & $\div$ & \\
\hline No trabajo & $\begin{array}{l}\text { Tipo de interacción mediante la } \\
\text { cual uno de los niños no trabaja, } \\
\text { aquí no hay interacción con el } \\
\text { compañero ni tampoco con la } \\
\text { tarea. El hecho de que el sujeto } \\
\text { no interactúe, se debe a que no se } \\
\text { encuentra conectado con la tarea. }\end{array}$ & 10 & \\
\hline
\end{tabular}

\section{Técnica de recolección de desempeños en pensamiento científico}

Para analizar el pensamiento científico en las diadas se utilizó el "índice de error", el cual es un indicador que da cuenta de la dificultad que representa para los niños llegar a resolver el problema. Se define como la suma de todos los errores que realiza cada diada durante la resolución de la tarea. Cuando el "índice de error" se aproxima o es igual a cero, significa que la diada tuvo éxito resolviendo la tarea; de esta manera, los índices de error más bajos significan un mejor desempeño en la tarea.

Para el juego Terapeia, un error equivale a que la diada envía una opción incorrecta de tratamiento o no envíe una opción correcta. De esta manera, si en el primer intento la diada envía un tratamiento incorrecto y uno correcto, pero omite otro correcto, obtendrá un índice de error de dos puntos; este proceso se repite con cada uno de los intentos de respuesta y se obtiene un índice de error total por sesión.

\section{Procedimiento}

La aplicación del juego tuvo una duración de tres meses, se llevaron a cabo nueve sesiones de juego (entre 20 y 30 minutos) una vez a la semana, con una semana de por medio. Durante cada sesión se obtuvo un puntaje de índice de error y los porcentajes de los tipos de interacción de cada diada.

De la sesión uno a la sesión tres se jugaron dos casos del nivel uno, un cuadro clínico del nivel dos y tres, cada cuadro clínico se compone de entre dos y tres casos. A partir de la sesión cuatro hasta la nueve, se jugaron la misma cantidad de cuadros clínicos del nivel dos y del nivel tres, pero ya no se realizó el nivel uno. Cada sesión se filmó a los participantes y se grabó la pantalla, usando el programa Atubechatcher (2017) versión 3.8.9 de libre acceso, el cual permitió capturar las sesiones de juego en su totalidad. Así pues, tres observadores siguieron el juego, teniendo cada uno una tarea específica. El primer observador, se encargó de realizar las filmaciones, el segundo acompañó a los niños mientras jugaron y el tercer observador llevó un registro donde su función fue anotar todas las observaciones y datos pertinentes para el análisis posterior de la investigación

Para asegurar la confiabilidad entre las observaciones de los tipos de interacción, se utilizó el estadístico de Kappa. Este método estadístico permitió tomar las observaciones de múltiples investigadores para, a través de repetidas codificaciones, obtener un dato con cierto nivel de concordancia. Para esta investigación se tomó un dato de interacción para cada sesión por pareja, para un total de 62 datos en las 9 sesiones, cada pareja pasó por una doble codificación y se encontró un porcentaje de concordancia promedio del 88\% en las diferentes diadas, lo cual equivale a un nivel fuerte (Carletta, 1996).

Al filmar todas las sesiones, se realizaron dos codificaciones por cada diada y por cada sesión, cada una realizada por un investigador diferente. Posteriormente se contrastaron los resultados obteniendo un puntaje final de la colaboración de las diadas. Al tener codificada todas las grabaciones se procedió a utilizar el programa Grideware (2018) versión 1.15 con el cual se crearon las gráficas de las trayectorias de interacción. 


\section{Resultados}

A continuación se presentarán los resultados obtenidos a través de las nueve sesiones aplicando el juego "terapeia” en las diadas. En una primera parte se presentaron las rejillas de estado que muestran la trayectoria de interacción de cada diada durante las sesiones para que, posteriormente, fuera posible realizar una comparación entre lo encontrado en las interacciones de cada diada y el desempeño en pensamiento científico que obtuvieron.
Para responder a la primera pregunta del estudio: ¿Cómo se presentan las trayectorias de interacción de diadas al resolver un problema científico a manera de videojuego?, se presentarán las rejillas de estado para cada diada.

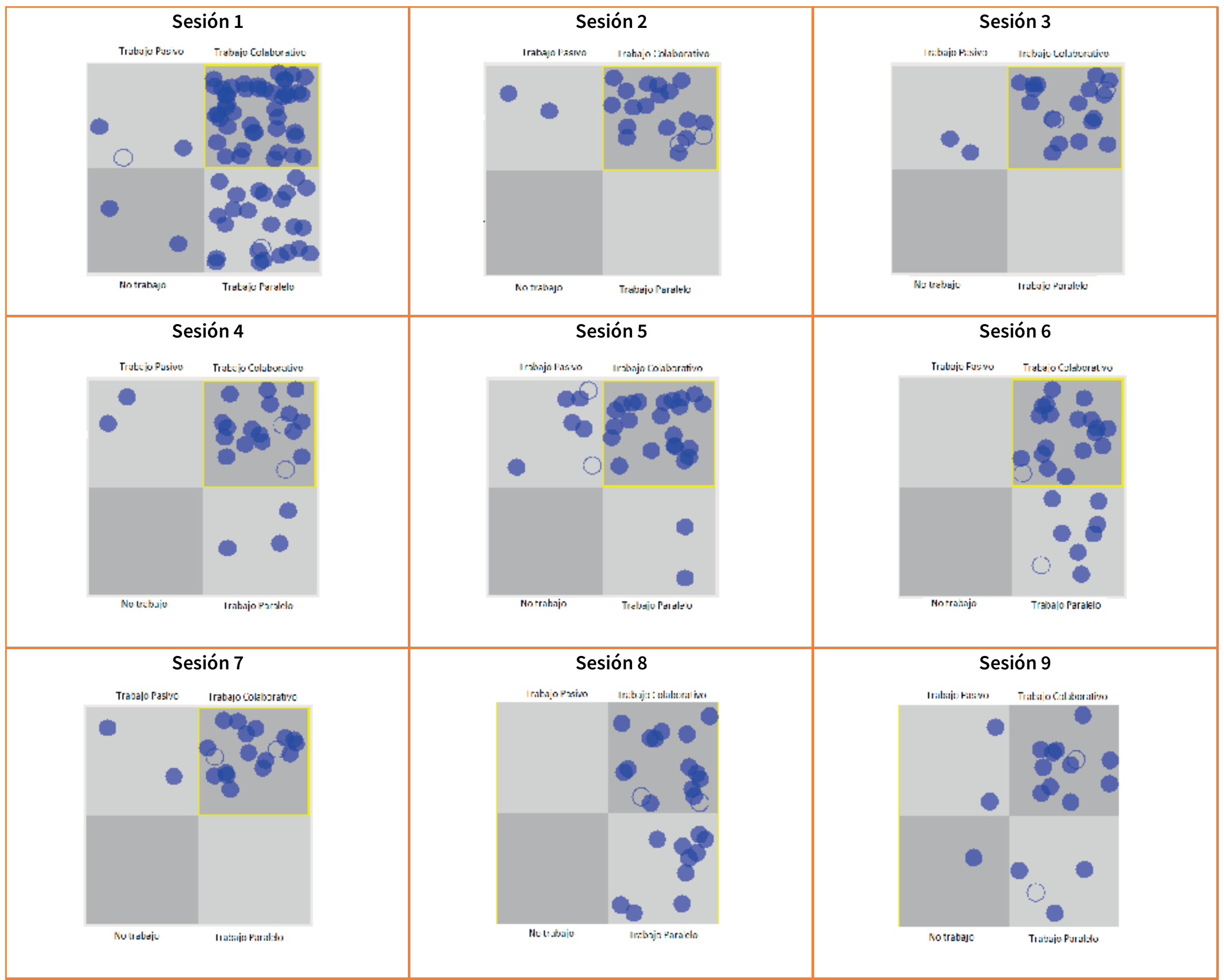

Figura 2 Resultado de las trayectorias de las interacciones de la diada 3 a lo largo de las nueve sesiones.

La diada 3, como se observa en la Figura 2, inicia con una colaboración del 60\%; en las dos siguientes sesiones incrementa significativamente hasta un 90\%, manteniéndose estable en la sesión dos y tres. Para la sesión cuatro disminuyen a un $\mathbf{7 8 \%}$, manteniéndose es table en las dos siguientes sesiones con un $71 \%$ y $70 \%$ respectivamente. En la sesión siete, la diada aumenta significativamente, obteniendo un porcentaje de 90\%, igual que en la sesión dos y tres. Para las últimas dos sesiones, la colaboración de esta diada disminuye y se mantiene en un rango de 63\% a 63\%. De esta manera, se puede decir que es una diada con tendencia a la colaboración, puesto que la mayor concentración de las interacciones se encuentra ubicadas en el trabajo colaborativo.

Con respecto a los demás tipos de interacción, se observa que la colaboración se presenta en menor medida. Sin embargo, durante la primera, sexta y séptima sesión las concentraciones del trabajo paralelo fueron de $\mathbf{3 3} \%$, $\mathbf{3 3} \%$ y $\mathbf{3 8} \%$ respectivamente. 


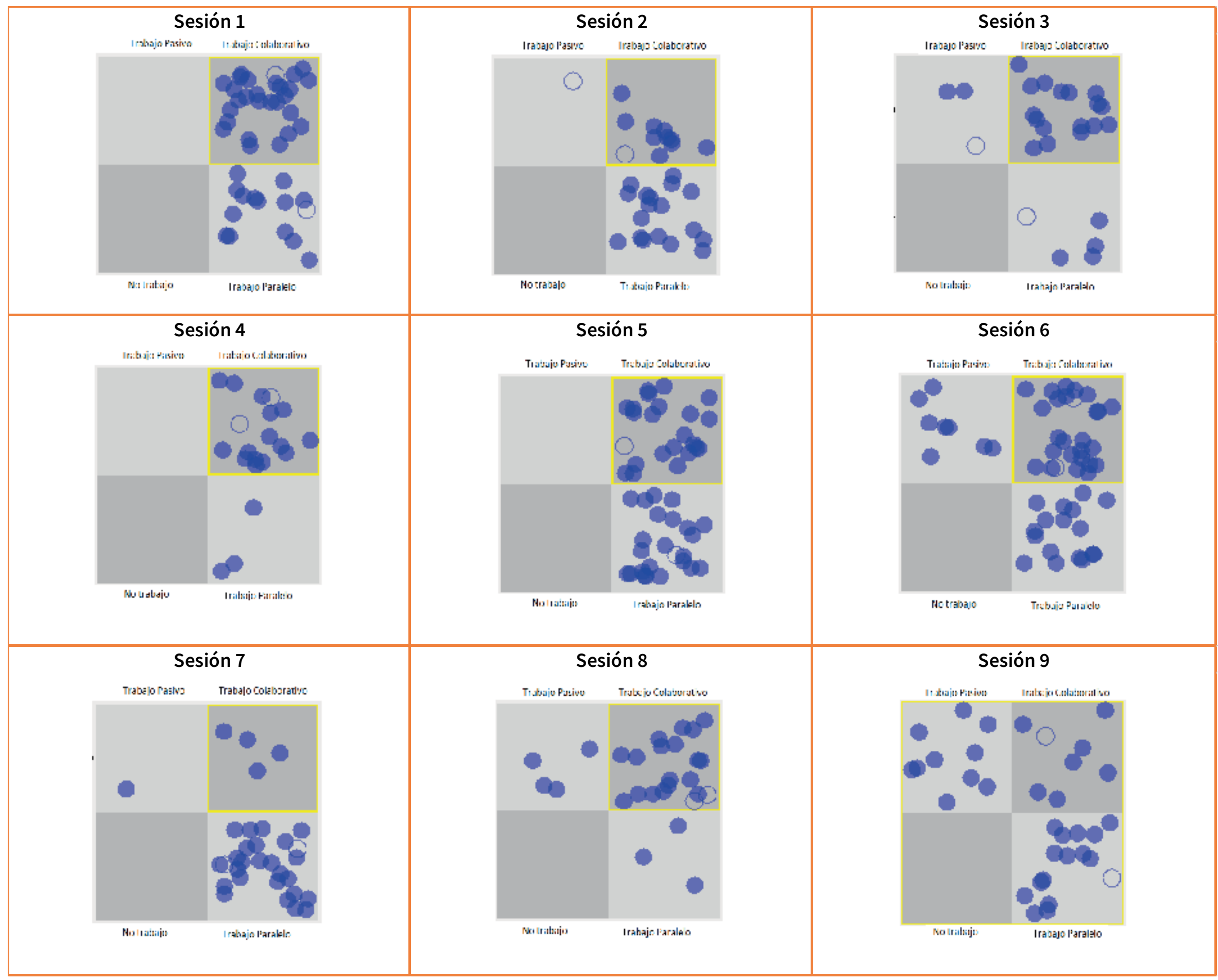

Figura 3. Resultado de las trayectorias de las interacciones de la diada 4 a lo largo de las nueve sesiones.

La diada 4, en la sesión uno, inició con un porcentaje de colaboración del 65\%. Después, en la segunda sesión la colaboración disminuyó a un 37\% y para la siguiente sesión volvió a tener una colaboración parecida a la de la primera con un 68\%. Posteriormente, en la siguiente sesión, subió significativamente a un 85\%, aunque para la sesión cinco, la colaboración volvió a disminuir a 49\%. En la sesión seis, subió moderadamente a 52\%, mientras que en la séptima sesión volvió a disminuir significativamente a 13\%. Aunque en la siguiente sesión logró aumentar a un 74\%, en la sesión final termino disminuyendo nuevamente hasta lograr una colaboración del 24\%.

Con respecto a su trabajo paralelo, se evidencian picos de aumento y disminución. Para la primera sesión, alcanzan un porcentaje de 35\%, luego en la sesión dos, está diada aumenta considerablemente presentando un 60\% en dicho tipo de trabajo. En la sesión cuatroy cinco, tienen una disminución considerable de 20\% y 15\%. Para la siguiente sesión, este tipo de trabajo vuelve a tener una mayor predominancia $51 \%$ y disminuye nuevamente en la siguiente sesión 33\%. En la séptima sesión, la diada aumentó de manera significativa sus interacciones de trabajo paralelo, alcanzando un porcentaje de 84\%. No obstante, para las últimas dos sesiones disminuyeron nuevamente, llegando, en la sesión ocho, a un 11\% y a un 47\% en la sesión nueve.

Dado a lo anterior, se puede evidenciar que esta diada presenta alta variabilidad en la concentración de sus datos, como se puede observar en la Figura 3. No obstante, la concentración de los datos se encuentra repartida principalmente entre el trabajo colaborativo y el trabajo paralelo. 


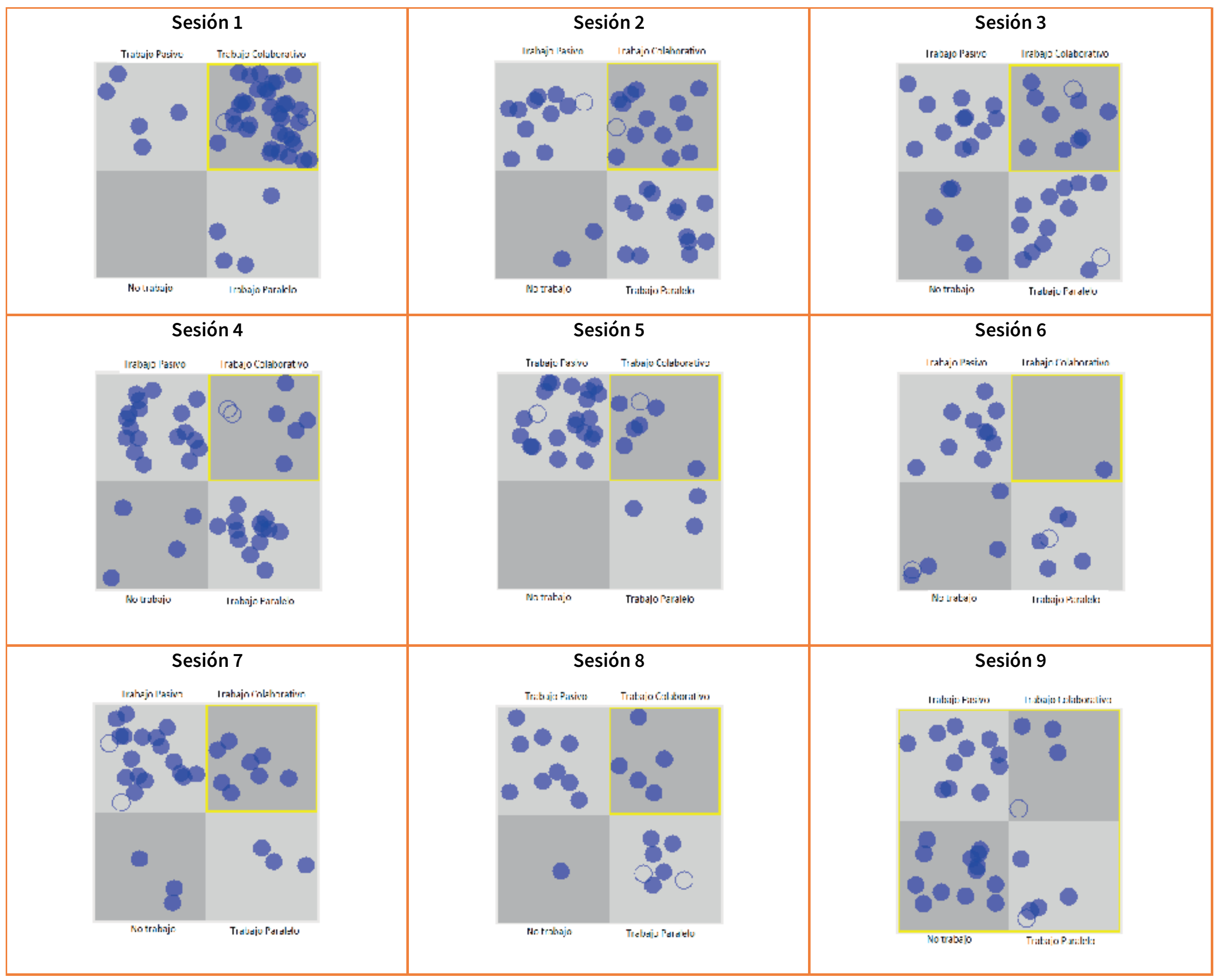

Figura 4. Resultado de las trayectorias de las interacciones de la diada 5 a lo largo de las nueve sesiones.

Con respecto a la diada 5 , se pudo observar un $\mathbf{8 0 \%}$ de colaboración al inicio. Sin embargo, en la segunda sesión disminuyó significativamente y se observó una colaboración del 35\%. Desde la segunda sesión, esta diada empezó a disminuir significativamente su colaboración hasta llegar a la sesión seis con un 5\%, aunque para la sesión siete y ocho aumentó un 10\% la colaboración, es decir, $\mathbf{2 4 \%}$ y $\mathbf{2 3} \%$ respectivamente. Por último, disminuyó una vez más, presentándose en la última sesión un 12\% de colaboración.

En cuanto a las interacciones del trabajo paralelo, la diada 5 no presenta aumentos significativos durante las sesiones, presentándose más constantes las interacciones de trabajo paralelo. En la sesión uno se registró un porcentaje de $\mathbf{9} \%$, mientras que durante las sesiones dos y tres se presentaron porcentajes del $\mathbf{3 3} \%$ y en la cuarta de $\mathbf{3 1} \%$. Para las sesiones cinco, seis y siete se presentó una disminución moderada 9\%, $27 \%$ y $9 \%$ consecutivamente. En la octava sesión, vuelve a tener un aumento de $\mathbf{3 2} \%$ para finalizar con una disminución leve de $\mathbf{1 5 \%}$.
Con relación al trabajo pasivo, el porcentaje durante las primeras cinco sesiones fue $\mathbf{1 1} \%, \mathbf{2 8} \%$, 30\%, $43 \%$ y $69 \%$ mostrado un aumento progresivo de este tipo de trabajo. En la sesión seis el porcentaje disminuyó a $\mathbf{4 5 \%}$ y volvió a aumentar en la sesión siete a $\mathbf{5 8 \%}$, aunque en las últimas dos sesiones disminuyó a $\mathbf{4 1 \%}$ y terminó en $\mathbf{3 3} \%$.

Con respecto a la concentración de los datos de esta diada, se pudo observar alta variabilidad en las interacciones; sin embargo, se presentó una tendencia al trabajo pasivo a medida que avanzaban las sesiones. 


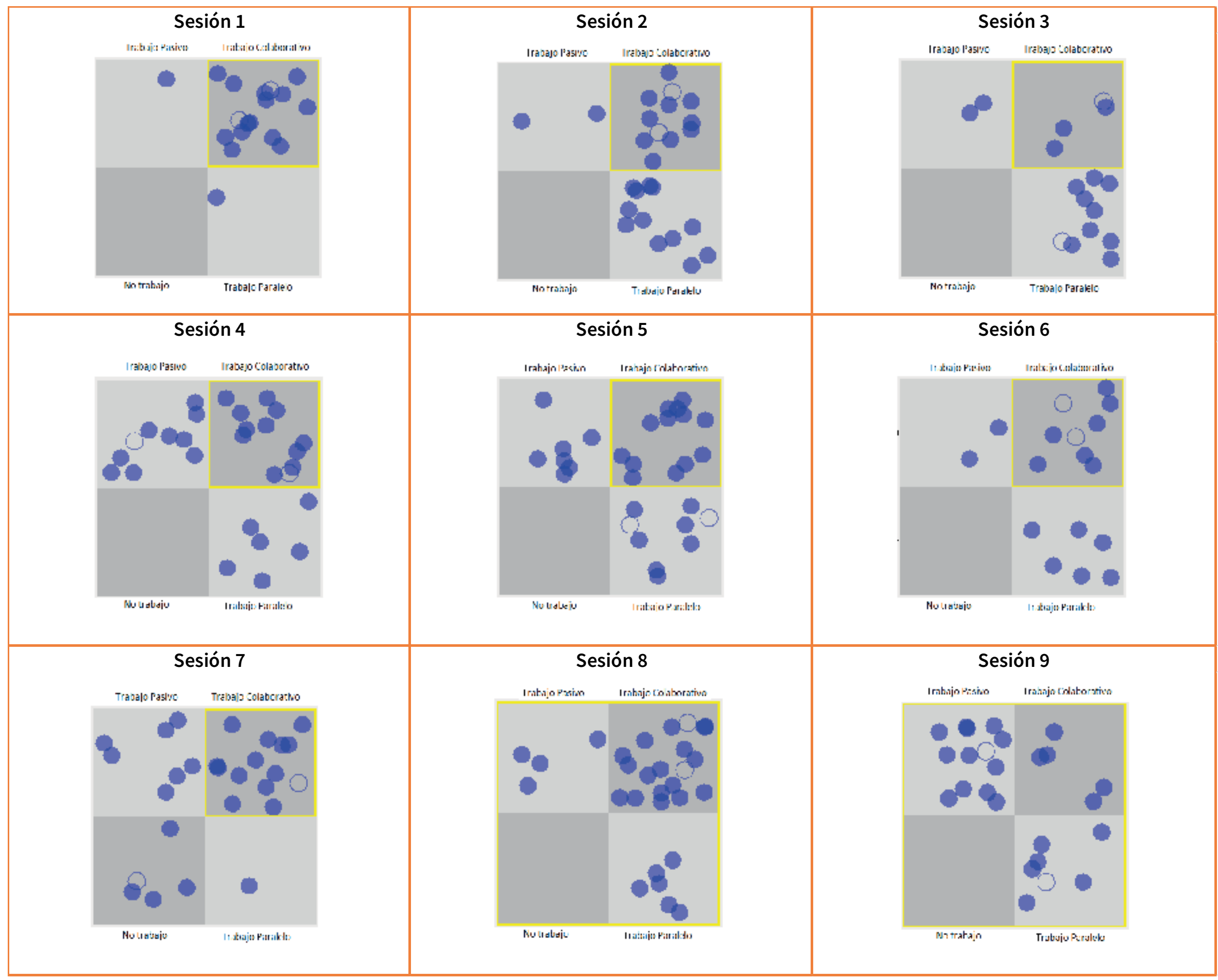

Figura 5. Resultado de las trayectorias de las interacciones de la diada 6 a lo largo de las nueve sesiones.

En cuanto a la diada 6 , se observó una colaboración inicial de un 89\%, para la segunda sesión, disminuye casi a la mitad, presentado una colaboración de 46\%. Para la tercera sesión, disminuye nuevamente y presenta un $25 \%$ en la colaboración. Para las siguientes sesiones, vuelve a aumentar la colaboración, quedando en un rango del 43\% al 45\%, similar a la segunda sesión. En la sesión seis y siete aumenta un 53\% y 52\% respectivamente, manteniéndose estable en estas dos sesiones. Para la siguiente sesión aumenta la colaboración con un 66\% y disminuye significativamente en la última sesión con un 20\%.

En cuanto al trabajo paralelo, este se presenta para la primera sesión con un porcentaje de 6\%, para la sesión dos y tres este aumenta considerablemente a 46\% y 63\%. En la sesión cuatro disminuyó a 21\% y nuevamente tuvo un aumento moderado en la sesión cinco y seis, quedando en un rango de 31\% y 35\%. Para la sesión siete, este tipo de trabajo vuelve a disminuir 4\%, siendo este similar a la primera sesión. Las dos últimas sesiones, nuevamente aumentan 21\% y 28\%.
Por otro lado, en el trabajo pasivo, durante las primeras sesiones se presentó un incremento progresivo que osciló entre el 6\%, 8\%, 13\% y $36 \%$, aunque en la sesión cinco y seis el trabajo pasivo disminuyó a 24\% y $12 \%$ respectivamente. Este tipo de trabajo aumenta en la sesión siete a 26\% y disminuyó una vez más en la sesión ocho a 14\%, terminando con un aumento significativo del 52\% en la última sesión. Con respecto a la concentración de los datos, se evidenció alta variabilidad en los diferentes tipos de trabajo en las primeras cuatro sesiones. En las sesiones siguientes, los datos se repartieron entre el trabajo pasivo, el trabajo colaborativo y el trabajo paralelo.

Ahora bien, una vez identificada la trayectoria de interacción de las diferentes diadas y para responder a la segunda pregunta (¿cuál es la relación entre las interacciones de los estudiantes al jugar en diadas y su desempeño durante múltiples sesiones al resolver un problema científico a manera de videojuego?), se presenta a continuación la relación entre el desempeño en pensamiento científico y las diferentes formas de interacción de cada diada. 
Tabla 2. Porcentaje de Colaboración e Índice de Error

\begin{tabular}{|c|c|c|c|c|c|c|c|c|c|c|}
\hline & Sesión & 1 & 2 & 3 & 4 & 5 & 6 & 7 & 8 & 9 \\
\hline \multirow{2}{*}{ Diada 3} & Colaboración & $60 \%$ & $90 \%$ & $90 \%$ & $78 \%$ & $71 \%$ & $70 \%$ & $90 \%$ & $62 \%$ & $63 \%$ \\
\hline & $\begin{array}{l}\text { Índice de } \\
\text { error }\end{array}$ & 100 & 63 & 25 & 33 & 60 & 53 & 29 & 48 & 32 \\
\hline \multirow{2}{*}{ Diada 4} & Colaboración & $65 \%$ & $37 \%$ & $68 \%$ & $85 \%$ & $49 \%$ & $52 \%$ & $13 \%$ & $74 \%$ & $24 \%$ \\
\hline & $\begin{array}{l}\text { Índice de } \\
\text { error }\end{array}$ & 106 & 67 & 48 & 28 & 119 & 108 & 64 & 53 & 57 \\
\hline \multirow{2}{*}{ Diada 5} & Colaboración & $80 \%$ & $35 \%$ & $25 \%$ & $17 \%$ & $22 \%$ & $5 \%$ & $24 \%$ & $23 \%$ & $12 \%$ \\
\hline & $\begin{array}{l}\text { Índice de } \\
\text { error }\end{array}$ & 99 & 86 & 78 & 60 & 57 & 33 & 62 & 29 & 59 \\
\hline \multirow{2}{*}{ Diada 6} & Colaboración & $89 \%$ & $46 \%$ & $25 \%$ & $43 \%$ & $45 \%$ & $53 \%$ & $52 \%$ & $66 \%$ & $20 \%$ \\
\hline & $\begin{array}{c}\text { Índice de } \\
\text { error }\end{array}$ & 41 & 43 & 28 & 42 & 54 & 28 & 44 & 45 & 43 \\
\hline
\end{tabular}

\section{Interacciones de la diada 3 y desempeño en pensamiento científico}

La diada 3 presentó una tendencia a la colaboración, como se puede observar en la Tabla 2. Además, las sesiones donde mayor porcentaje de colaboración se presentó fueron las de mejor desempeño en pensamiento científico. Si bien las primeras sesiones mostraron los porcentajes de colaboración más altos acompañados de una disminución progresiva del índice de error que obtenían, más adelante la colaboración tendió a estabilizarse. Además, con relación al índice de error, la diada presentó una pendiente negativa de -4.8, es decir, que se presentó una disminución general del índice de error a lo largo de las sesiones.

Durante las interacciones, los estudiantes de esta diada mostraron constante interés por escuchar las ideas de su compañero, en la mayoría de los casos se realizaban preguntas entre sí o hacían comentarios para asegurarse que ambos estuvieran pensando lo mismo. Además, en algunas ocasiones donde se encontraban en desacuerdo se tomaban el tiempo para explicarle el uno al otro porque pensaban que un tratamiento determinado iba a ser efectivo.

\section{Interacciones de la diada 4 y desempeño en pensamiento científico}

La relación entre las interacciones y el desempeño en pensamiento científico de esta diada da cuenta de gran variabilidad en las diferentes estrategias empleadas para resolver la tarea. Se observó que el desempeño de esta diada es tan variable como las diferentes estrategias que usan. De esta manera, aunque se presentan sesiones donde mejora el desempeño en pensamiento científico y aumenta el trabajo colaborativo, también se evidencian sesiones de buen desempeño y trabajo paralelo alto. Con respecto al índice de error, este disminuyó de forma general presentando una pendiente negativa de -2.1 .

Durante la interacción con el juego, esta diada presentó sesiones de altos niveles de colaboración, se escuchaban el uno al otro y esperaban hasta llegar a acuerdos antes de enviar opciones de tratamiento. Del mismo modo, mostraron interacciones de alto trabajo paralelo donde, aun cuando ambos estudiantes estuvieran enfocados en superar la tarea, seguían sus propias ideas y deseos a la hora de enviar opciones de tratamiento pero sin detenerse a discutir como grupo; en algunas ocasiones se observó que se turnaban para jugar.

\section{Interacciones de la diada 5 y desempeño en pensamiento científico}

Con respecto a las relaciones entre el desempeño de los participantes y las interacciones se observó que la diada 5 presentó una tendencia al trabajo pasivo y al mismo tiempo una mejora en el desempeño de pensamiento científico a lo largo de las sesiones. Sin embargo, se presentaron sesiones con diferentes tipos de interacción donde el desempeño mejoró. Además, se observó una tendencia en la que el desempeño y el porcentaje de colaboración disminuyeron proporcionalmente, es decir que tendieron a mejorar su desempeño trabajando individualmente. Por otro lado, la pendiente del índice de error de esta diada fue de -6.5, es decir que presentaron una disminución del error de manera general a lo largo de las sesiones.

Durante la interacción con el videojuego, generalmente, había uno de los participantes que tomaba el mando de la sesión mientras el otro lo observaba; sin embargo, no siempre fue el mismo estudiante al mando durante todas las sesiones, ni lo tenía durante toda la sesión, aunque fueron pocas las ocasiones donde ambos lograban ponerse de acuerdo a la hora de enviar una opción de tratamiento. En algunas ocasiones, incluso, uno de los estudiantes ignoraba la opinión de su compañero, aunque esta pudiera ser correcta. 


\section{Interacciones de la diada 6 y desempeño en pensamiento científico}

Con respecto a la relación entre el desempeño en pensamiento científico y las interacciones de esta diada, se puede observar que en la mayoría de las sesiones el primero se mantiene estable en un rango de 41 a 45, mientras que la concentración de los diferentes tipos de interacción varía durante las distintas sesiones; en algunas predomina un tipo de trabajo, mientras que en otras se reparten equitativamente, sin observarse relación con el desempeño. Lo anterior da cuenta de la variabilidad de esta diada. Además, la pendiente de esta diada fue de 0.53 es decir que a lo largo de las sesiones el índice de error presentó un leve incremento.

Durante la aplicación del videojuego, esta diada exploró diferentes formas de interacción entre las sesiones; incluso, en muchas ocasiones en una misma sesión empezaban con un tipo en particular de interacción, pero a medida que avanzaba el juego, cambiaban su estrategia.

\section{Discusión}

Para comenzar se identificó que, a pesar de la variabilidad de las trayectorias de colaboración y desempeño, se lograron observar algunas tendencias en la interacción de las diadas, lo que permitió realizar una categorización. Dentro de lo observado, durante las nueve sesiones, aparecieron dos tendencias: la primera es que algunas díadas que se pueden denominar como "organizadas" tendían a un tipo de interacción entre los cuatro posibles. En este punto se pudo identificar una diada con propensión a la colaboración y una diada con una propensión al trabajo pasivo. Con respecto a la segunda tendencia, se observaron diadas cuya estructura fue menos "organizada". Es decir, que esas díadas fueron altamente variables y probaron los diferentes tipos de interacciones a lo largo de las sesiones.

Contrario al estudio de Guevara, Dijk, \& Geert (2016), quien encontró que, durante la interacción de diadas en la tarea de resolución de problemas, el trabajo paralelo predominó sobre las demás formas de interacción en los participantes, en esta investigación ninguna de las diadas mostró una tendencia hacia el trabajo paralelo, sino hacia trabajo colaborativo en la diada 3, hacia el trabajo pasivo en la diada 5 y alta variabilidad en las interacciones en las demás diadas. Este hallazgo se podría explicar debido a las diferencias en la naturaleza del videojuego y a la edad de los niños, quienes tenían entre cuatro y seis años.

Al analizar la relación entre las interacciones de las diadas y su desempeño en la resolución de la tarea, se observó que las diadas cuya estructura fue más organizada mostraron mejoría en el desempeño de la tarea. Esto quiere decir que no solamente el grupo que colabora es el que podrá tener mejor desempeño, sino que los grupos donde existan roles bien definidos y se realice un trabajo organizado también lo pueden presentar.

Del mismo modo, al analizar lo encontrado con las pendientes de las diadas se evidencia el mismo resultado en el desempeño ya que, al comparar las pendientes de cada diada, los puntajes más bajos que significan una mayor mejora en el desempeño en la tarea a lo largo de las nueve sesiones lo obtienen la diada 5 con una pendiente de - 6.5 y la diada 3 con -4.8. La diada 4 aunque también presentó un puntaje negativo, fue menor en las diadas anteriores.
Estos hallazgos matizan los planteamientos de autores como Kuhn (2015), Sánchez, Mendoza, \& Salinas (2009) y Sung \& Hwang (2013) quienes encontraron que, durante la interacción entre pares, el desempeño en una tarea tenía mejoras significativas, cuando estas colaboraban e interactuaban activamente entre sí. Lo encontrado en este estudio da cuenta de que, aunque la colaboración puede ser una condición que favorece la mejora en el desempeño, no es la única forma de interacción que lo permite. Esto se evidencia con claridad en la diada 5, que mostró una mejoría en su desempeño a lo largo de las sesiones y su estructura de interacción tendió al trabajo pasivo.

La afirmación anterior, sobre la relación entre los tipos de interacción y el desempeño, fue debatida por Fawcett y Garton (2005), quienes encontraron que los efectos de la colaboración en niños de seis y siete años dependían, en cierta medida, de la competencia que presentaran los participantes. Así, los estudiantes que más se vieron beneficiados por la colaboración fueron los menos hábiles, en comparación con los que demostraron mayor habilidad.

Lo anterior tiene relación con los hallazgos de esta investigación, puesto que apoya la idea de la colaboración como un fenómeno variable y que, aunque puede llegar a ser una condición favorable para el desarrollo de habilidades o la mejora en el desempeño de una tarea, no es la única razón que puede incidir en estos aspectos. De esta manera, se podrían tener en cuenta aspectos como la estructura de la interacción, es decir, la forma como la diada se organiza para enfrentarse a la tarea ya que, cuando un sistema se organiza con roles definidos y se distribuyen tareas para lograr un objetivo, es posible que se presente la mejora en el desempeño de la actividad. Sin embargo, en este orden de ideas, también es posible que la díada se beneficie de forma diferenciada de la interacción con su compañero y con el videojuego.

Cabe resaltar que la naturaleza de la tarea es otra variable a tener en cuenta al hablar de los cambios en el desempeño. Estudios de diferentes autores (Castillo, Checa, García-Varela, Herrero, \& Monjelat, 2014; Papastergiou, 2009; Annetta, Minogue, Holmes, \& Cheng, 2009; Brom, Preuss, \& Klement, 2011; Fengfeng, 2008); han encontrado que los videojuegos influyen en el desarrollo de diferentes habilidades, procesos de pensamiento o en la adquisición de conocimiento. Sin embargo, este estudio da cuenta de que el fenómeno es mucho más complejo, los videojuegos o la interacción por si solos no generan el desarrollo de lo mencionado anteriormente. Por el contrario, el desarrollo del razonamiento científico sería un producto emergente de la relación entre las características del videojuego, el tipo y organización de las interacciones de las diadas y probablemente otras variables como el conocimiento previo, aspectos motivacionales, andamiaje y otros aspectos que por ahora escapan al alcance de este texto. Lo anterior es todavía más relevante si se tiene en cuenta que el contexto en el que los niños juegan con videojuegos es generalmente complejo y tiene altos componentes sociales, lo que no siempre ocurre en contextos educativos.

Ahora bien, el abordaje que presentó esta investigación como lo plantea Guevara, Dijk, \& Geert (2016), permitió develar los procesos que ocurren en tiempo real en las diadas, estudiar la naturaleza variable del desarrollo e identificar patrones y tendencias que otros abordajes no. Lo anterior se sustenta al observar la naturaleza de los resultados obtenidos y el análisis realizado, los cuales permiten comprender la naturaleza variable y multicausal de emplear herramientas como los videojuegos. 
Además, permitió tomar distancia de la perspectiva tradicional donde los resultados se limitan a identificar el efecto que tienen o no los videojuegos sobre los participantes (Castillo, Checa, García-Varela, Herrero, \& Monjelat, 2014; Papastergiou, 2009; Annetta, Minogue, Holmes, \& Cheng, 2009; Brom, Preuss, \& Klement, 2011; Fengfeng, 2008; Lacasa, Martínez, \& Méndez, 2008) y comprender algunas de las variables que entran a interactuar en las diadas para generar cambios en el desempeño.

\section{Conclusiones}

A manera de conclusión, partiendo de lo encontrado en relación a la naturaleza dinámica y variable de la colaboración durante la aplicación de un videojuego se podría decir que, en un contexto escolar es necesario tener en cuenta no solamente la colaboración como estrategia para fomentar el desarrollo en los estudiantes. Lo observado da cuenta que la relación entre la mejora del desempeño no va relacionada únicamente a la colaboración, sino que otra variable que entra a interactuar es lo definido de la estrategia empleada, es decir, que mientras haya una estructura y un orden dentro de los equipos de trabajo, también es posible pensar en una mejora del desempeño.

De esta manera, específicamente hablando de la colaboración, se puede concluir que es un fenómeno que no aparece permanentemente durante el trabajo en grupo, sino que oscila en diferentes periodos de tiempo. Por tal razón, no es posible pensar que en un contexto educativo formal se les exija a los estudiantes trabajar de manera colaborativa durante todo el tiempo de la actividad. Por esta razón, es necesario propiciar nuevas estrategias que permitan la flexibilidad del sistema, que les posibiliten a los estudiantes explorar estrategias distintas a la colaboración.

Teniendo en cuenta lo anterior, los videojuegos podrían ser una de estas estrategias, ya que funcionan como un escenario que por sí mismos y sus características, permiten la aparición de procesos que van más allá de la interacción de un niño frente a una situación de resolución de problemas, favorece la puesta en marcha de herramientas de pensamiento científico (Kuhn, Lordanou, Pease, \& Wirkala, 2008). Esta investigación tal como lo plantea el juego Terapeia, se caracteriza por presentar durante cada sesión una problemática compuesta por diversos casos, que pese a ser isomorfos (tener una estructura similar), presenta diferentes niveles de dificultad con problemáticas distintas. Estas características, hacen que este sea un escenario cambiante, lo cual facilita la flexibilidad y emergencia de las múltiples formas de interacción en los jugadores.

Por esta razón, la propuesta de usar videojuegos educativos en contextos escolares, debe partir de la comprensión de estas herramientas no como generadoras de desarrollo, sino como herramientas que contribuyen a la aparición de las variables que pueden posibilitar la mejora del desempeño en los estudiantes. Sin embargo, para involucrar los videojuegos es necesario tener en cuenta la necesidad del acompañamiento de una instrucción y del apoyo del docente que guíe y oriente a los estudiantes mientras los usan.

Una de las limitaciones de este estudio radica en la afirmación anterior, puesto que, aunque se trató de observar el fenómeno en un ambiente "naturalizado", la verdadera naturaleza de un contexto educativo formal, está atravesada por múltiples variables como la presencia del maestro, la necesidad de seguir un currículo académico, la presencia de los demás estudiantes en los salones de clase, entre otros.

\section{Referencias}

Anderson, A., Brunner, C., Culp, K., Diamond, J., Lewis, A., \& Martin, W. (2009). Using microgenetic methods to investigate problem solving in video games. In Digital Games Research Association (DiGRA) Conference, 5, 1-7. Obtenido de http://www.digra.org/digital-library/publications/using-microgeneticmethods-to-investigate-problem-solving-in-video-games/?doing wp cron=1 $\underline{543955824.3273510932922363281250}$

Anderson, J., \& Barnett, M. (2013). Learning physics with digital game simulation in middle school science. Journal of science education and technology, 22(6), 914-926. doi:10.1007/s10956-013-9438-8

Annetta, L., Minogue, J., Holmes, S., \& Cheng, M. (2009). Investigating the impact of video games on high school students' engagement and learning about genetics. Computers and education, 53(1), 74-85. doi:https://doi. org/10.1016/j.compedu.2008.12.020

Barab, S., Scott, B., Siyahhan, S., Goldstone, R., Ingram-Goble, A., Zuiker, S., \& Warren, S. (2009). Transformational play as a curricular scaffold: Using videogames to support science education. Journal of Science Education and Technology, 18(4), 305-320. Obtenido de https://link.springer.com/ article/10.1007/s10956-009-9171-5

Bermejo, V. (2005). Microgénesis y cambio cognitivo: adquisición del cardinal numérico. Psicothema, 17(4), 559-562. Obtenido de http://www.psicothema. com/psicothema.asp?id=3145

Brom, C., Preuss, M., \& Klement, D. (2011). Are educational computer micro-games engaging and effective for knowledge acquisition at high-schools? A quasiexperimental study. Computers and education, 57(3), 1971-1988. doi:https:// doi.org/10.1016/j.compedu.2011.04.007

Carletta, J. (1996). Assessing Agreement on Classification Tasks: The Kappa Statistic. Journal Computational Linguistics, 22(2), 249-254. Obtenido de https://dl.acm.org/citation.cfm?id=230390

Castillo, H., Checa, M., García-Varela, A., Herrero, D., \& Monjelat, N. (2014). Evolution and natural selection: learning by playing and reflecting. Journal of New Approaches in Educational Research, 3(1), 1-26. doi:DOl: 10.7821/naer.3.1.26$\underline{33}$

Cimpian, A. (2010). The impact of generic language about ability on children's achievement motivation. Developmental Psychology, 46(5), 1333-1340. doi:http://dx.doi.org/10.1037/a0019665

Clark, D., Nelson, B., Chang, H., Martinez-Garza, M., Slack, K., \& D’Angelo, C. (2011). Exploring Newtonian mechanics in a conceptually-integrated digital game: Comparison of learning and affective outcomes for students in Taiwan and the United States. Computers y Education, 57(3), 2178-2195. doi:https://doi. org/10.1016/j.compedu.2011.05.007

Corredor, J., Gaydos, M., \& Squire, K. (2013). Seeing Change in Time: Video Games to Teach about Temporal Change in Scientific Phenomena. Journal Of Science Education And Technology, 23(3), 324-343. Obtenido de https://link. springer.com/article/10.1007/s10956-013-9466-4

Csikszentmihalyi, M. (2008). Flow: the psychology of optimal experience. New York: Harper Perennial. Obtenido de https://www.harpercollins. ca/9780061339202/flow/

Fawcett, L., \& Garton, A. (2005). The effect of peer collaboration on children's problem凶solving ability. British Journal of Educational Psychology, 75(2), 157-169. doi:https://doi.org/10.1348/000709904X23411

Fengfeng, K. (2008). A case study of computer gaming for math: Engaged learning from gameplay? Computers and education, 51(4), 1609-1620. doi:https://doi. org/10.1016/j.compedu.2008.03.003

Flavell, J. (1979). Metacognition and cognitive monitoring: A new area of cognitivedevelopmental inquiry. American Psychologist, 34(10), 906-911. doi:http:// dx.doi.org/10.1037/0003-066X.34.10.906

Flavell, J., Miller, P., \& Miller, S. (2002). Cognitive Development. New York: Englewood Cliffs. Obtenido de https://www.pearson.com/ us/higher-education/product/Flavell-Cognitive-Development-3rdEdition/9780131400399.html

Garcia- Ruiz, M., \& Orozco, L. (2008). Orientando un cambio de actitud hacia las Ciencias Naturales y su enseñanza en profesores de educación primaria. Revista Electrónica de Enseñanza de las Ciencias, 7(3), 1-30. Obtenido de http://reec.uvigo.es/volumenes/volumen7/ART3 Vol7 N3.pdf

Golbeck, S. (1998). Peer collaboration and children's representation of the horizontal surface of liquid. Journal of Applied Developmental Psychology, 19(4), 571-592. doi:https://doi.org/10.1016/S0193-3973(99)80056-2 
Guevara, M., Dijk, M., \& Geert, P. (2016). Microdevelopment of peer interactions and scientific reasoning in young children. Journal for the Study of Education and Development, 39(4), 727-771. doi:https://doi.org/10.1080/02103702.2016. $\underline{1215083}$

Herrero, D., Monjelat, N., García-Varela, A., Checa, M., \& Gómez, P. (2014). Evolution and natural selection: learning by playing and reflecting. Journal of New Approaches in Educational Research, 3(1). doi:https://doi.org/10.7821/ naer.3.1.26-33

Holbert, N., \& Wilensky, U. (s.f.). Constructible authentic representations: Designing video games that enable players to utilize knowledge developed in-game to reason about science. Technology, Knowledge and Learning, 19(1), 53-79. doi:https://doi.org/10.1007/s10758-014-9214-8

Hollenstein, T. (2007). State space grids: Analyzing dynamics across development. International Journal of Behavioral Development., 31(4), 384-396. doi:DOI: 10.1177/0165025407077765

Instituto Colombiano para el Fomento de la Educación Superior. (24 de Octubre de 2016). Estudiantes de colegios oficiales mueven positivamente el examen Saber $11^{\circ}$. Obtenido de Icfes mejor saber.: http://www2.icfes.gov.co/ item/2117-estudiantes-de-colegios-oficiales-mueven-positivamente-el-e

Kiili, K., de Freitas, S., Arnab, S., \& Lainema, T. (2012). The design principles for flow experience in educational games. Procedia Computer Science, 15, 78-91. doi:https://doi.org/10.1016/j.procs.2012.10.060

Kim, B., Park, H., \& Baek, Y. (2009). Not just fun, but serious strategies: Using metacognitive strategies in game-based learning. Computers and Education, 52(4), 800-810. doi:https://doi.org/10.1016/j.compedu.2008.12.004

Ko, S. (2002). An Empirical Analysis of Children's Thinking and Learning in a Computer Game Context. Educational Psychology, 22(2), 219-233. doi:https://doi.org/10.1080/01443410120115274

Koriat, A. (2012). When Two Heads Are Better Than One and When They Can Be Worse: The Amplification Hypothesis. Journal of Experimental Psychology: General. American Psychological Association, 144(5), 934 -950. doi:http:// dx.doi.org/10.1037/xge0000092

Kuhn, D. (2015). Thinking togheter and alone. Educational Researcher, 44, 46-53. doi:https://doi.org/10.3102/0013189X15569530

Kuhn, D., Lordanou, K., Pease, M., \& Wirkala, C. (2008). Beyond control of variables: What needs to develop to achieve skilled scientific thinking? Cognitive Development, 23(4), 435-451. doi:https://doi.org/10.1016/j. cogdev.2008.09.006

Lacasa, P., Martínez, R., \& Méndez, L. (2008). Developing new literacies using commercial videogames as educational tools. Linguistics and education, 19(2), 85-106. doi:DOI: 10.1016/j.linged.2008.02.001

Martí, E. (2003). Representar el Mundo Externamente. Madrid: Aprendizaje. Obtenido de https://www.casadellibro.com/libro-representar-el-mundoexternamente-la-adquisicion-infantil-de-los-sistemas-externos-de-representa cion/9788477741435/928751

McGonigal, J. (2011). Reality is broken: Why games make us better and how they can change the world. New York: Penguin. Obtenido de https://WwW. amazon.es/Reality-Broken-Games-Better-Change/dp/0143120611

McMillan, J., \& Schumacher, S. (2005). Diseños de investigación no experimental y encuestas. Madrid: Pearson.

Morris, B., Croker, S., Zimmerman, C., Gill, D., \& Roming, C. (2013). Gaming science: the "gamification" of science thinking. Frontiers in psychology, 4(607), 1-16. doi:https://doi.org/10.3389/fpsyg.2013.00607

Mullins, D., Rummel, N., \& Spada, H. (2011). Are two heads always better than one? Differential effects of collaboration on students' computer-supported learning in mathematics. International Journal of Computer-Supported Collaborative Learning, 6(3), 421-443. doi:http://dx.doi.org/10.1007/s11412011-9122-z

Neulight, N., Kafai, Y., Kao, L., Foley, B., \& Galas, C. (2007). Children's participation in a virtual epidemic in the science classroom: Making connections to natural infectious diseases. Journal of Science Education and Technology, 16(1), 47-58. doi:DOI: 10.1007/s10956-006-9029-z

Ocelli, M., Biber, P., Willging, P., \& Valeiras, N. (2015). Jugary aprender biología celular: una experiencia con el videojuego Kokori. . XI Jornadas Nacionales y VI Congreso Internacional de Enseñanza de la Biología. General Roca. dol:DOl: 10.13140/2.1.4881.7603

Organización de las Naciones Unidas . (2015). Organización de las Naciones Unidas. Obtenido de Educación para todos: http://www.unesco.org/new/es/ our-priorities/education-for-all/,
Organización de las Naciones Unidas para la Educación las Naciones Unidas para la Educación, I. C. (2017). Objetivos de desarrollo sostenible. . Paris: UNESCO. Obtenido de http://unesdoc.unesco.org/ images/0025/002524/252423s.pdf

Papastergiou, M. (2009). Digital Game-Based Learning in high school Computer Science education: Impact on educational effectiveness and student motivation, . Journal Computers and education , 52(2), 1-12. doi:https://doi. org/10.1016/j.compedu.2008.06.004

Park, J., \& Lee, J. (2015). Dyadic Collaboration Among Preschool-Age Children and the Benefits of Working With a More Socially Advanced Peer. Journal Early Education and Development, 26(4), 574-593. doi: https://doi.org/10.1080/104 09289.2015.995567

Plass, J., O’Keefe, P., Homer, B., Case, J., Hayward, E., Stein, M., \& Perlin, K. (2013). The Impact of Individual, Competitive, and Collaborative Mathematics Game Play on Learning, Performance, and Motivation. . Journal of Educational Psychology, 105(4), 1050-1066. doi:http://dx.doi.org/10.1037/ a0032688

Przybylski, A., Ryan, R., \& Rigby, S. C. (2009). The motivating role of violence in video games. . Personality and Social Psychology Bulletin, 35, 243-259. doi:https://doi.org/10.1177/0146167208327216

Quintanal Pérez, F. (2016). Aplicación de herramientas de gamificación en física y química de secundaria. Revista Opcion, 32(12), 327-348. Obtenido de https://www.researchgate.net/publication/311767939 Aplicacion de herramientas de gamificacion en fisica_y quimica de secundaria

Ritterfeld, U., \& Weber, R. (2006). Video games for entertainment and education. . En P. Vorderer, \& J. Bryant, Playing video games: Motives, responses, and consequences. (págs. 399-413). Mahwah, New Jersey: Routledge.

Sánchez, J., Mendoza, C., \& Salinas, A. (2009). Mobile serious games for collaborative problem solving. Journal Studies in Health Technology and Informatics., 193-197. doi:doi:10.3233/978-160750-017-9193

Schulze, J., Martin, R., Finger, A., Henzen, C., Lindner, M., Pietzsch, K., ... Zander, U. (2015). Design, implementation and test of a serious online game for exploring complex relationships of sustainable land management and human well-being. Journal Environmental Modelling and Software, 65, 58-66. doi:https://doi.org/10.1016/j.envsoft.2014.11.029.

Shernoff, D., Csikszentmihalyi, M., Schneider, B., \& Steele, E. (2003). Student Engagement in High School Classrooms from the Perspective of Flow Theory. . Journal School Psychology Quarterly , 18(2), 158-176. doi:https:// doi.org/10.1007/978-94-017-9094-9

Shernoff, D., Hamari, J., \& Rowe, E. (Junio de 2014). Edmedia . Obtenido de Edmedia : https://www.researchgate.net/publication/264046054 Measuring_Flow in Educational_Games and Gamified Learning Environments

Shute, V., \& Kim, Y. (2012). Does playing the World of Goo facilitate learning? . En D. Dai, Design research on learning and thinking in educational settings: Enhancing intellectual growth and functioning (págs. 243-267). New York: Routledge Books. Obtenido de https://books. google.com.co/books? id=iJZdBWAAQBAJ\&lpg=PPI\&dq=Shute\%2C\%20 v.\%20y\%20Kim\%2C\%20Y.\%20(2012)\%20Does\%20playing\%20the\%20 World\%200f\%20Goo\%20facilitate\%20learning\%3F\%20Dai\%2C\%20 D.\%20(Ed.)\%2C\%20Design\%20research\%20on\%20learning\%20and\%20 thinking\%20in\%20educational\%20settings\%3A\%20\%20Enhancing\%20 intell\& $\mid r \& h l=e s \& p g=P P 1 \# v=0 n e p a g e \& q \& f=$ false

Siegler, R. (1994). Cognitive Variability: A key to Understanding Cognitive Development. Current Directions in Psychological Science, 3(1), 1-5. doi:http://dx.doi.org/10.1111/1467-8721.ep10769817

Sills, J., Rowse, G., \& Emerson, L. M. (2016). The role of collaboration in the cognitive development of young children: a systematic review. . Journal child: Care, Health and Development, 42, 313-324. doi:https://doi. org/10.1111/cch.12330

Squire, K. (2008). Video game-based learning: An emerging paradigm for instruction. . Journal Performance Improvement Quarterly, 21(2), 7-36. doi:https://doi.org/10.1002/piq.20020

Squire, K., \& Jan, M. (2007). Mad City Mystery: Developing Scientific Argumentation Skills with a Place-based Augmented Reality Game on Handheld Computers. . Journal of Science Education and Technology, 16(1), 5-29. doi:https://doi.org/10.1007/s10956-006-9037-z

Sung, H.-Y., \& Hwang, G.-J. (2013). A collaborative game-based learning approach to improving students' learning performance in science courses. Journal Computers and education, 63, 43-51. doi:https://doi.org/10.1016/j. compedu.2012.11.019 
Torrance, H. (2007). Assessment as learning? How the use of

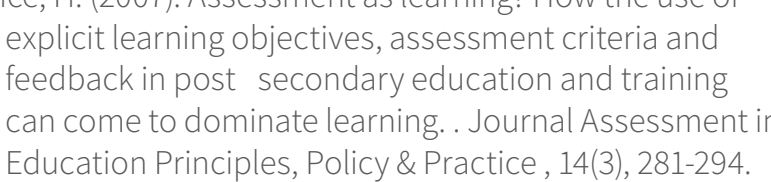

solving and learning motivation. Journal Computers and

ducation Principles, Policy \& Practice, 14(3), 281-294.

. . . . Chen. 2012.01.012

Ya-Tin, C. Y. (2012). Building virtual cities, inspiring intelligent
citizens: Digital games for developing students' problem

social capital. 20, 1-36. Obtenido de Communications 